\section{Commentary: Simple and effective subvalvular repair for ischemic mitral regurgitation: Yes, we can!}

\author{
Michael Silverman, MD, ${ }^{\mathrm{a}}$ and \\ Muralidhar Padala, $\mathrm{PhD}^{\mathrm{a}, \mathrm{b}}$
}

Ischemic mitral regurgitation (IMR), the subtype of mitral regurgitation that results from chronic changes to left ventricular structure and function from cardiomyopathies, is an independent predictor of cardiovascular morbidity and mortality. ${ }^{1}$ Treatment begins with guideline-directed medical therapy to reduce the severity of regurgitation, by altering the hemodynamic parameters, by reversing the left ventricular dysfunction, or by elevating the valveclosing forces. A variety of other options exist, including cardiac resynchronization therapy, transcatheter valve repair, and surgical valve repair, which address either the ventricle or the valve directly, with an end goal to correct the regurgitation and remove volume overload on the dysfunctional left ventricle. Nevertheless, the optimal treatment plan beyond medical therapy and cardiac resynchronization therapy remain a subject of debate, and the efficacy of either is limited to a select set of patients. ${ }^{2}$

Among direct valvular interventions, surgical repair or replacement is the traditional approach, and transcatheter interventions are emerging. Although the latter is increasingly replacing surgery as the first choice, in patients undergoing revascularization with coronary artery bypass grafting, surgical correction of IMR is the preferred option. In these patients, whether surgical valve replacement is a better choice than repair continues to be a debate, but it is

From the a Structural Heart Research and Innovation Laboratory, Carlyle Fraser Heart Center at Emory University Hospital Midtown, Atlanta, Ga; ${ }^{\mathrm{b}}$ Division of Cardiothoracic Surgery, Emory University School of Medicine, Atlanta, Ga.

Disclosures: The authors reported no conflicts of interest.

The Journal policy requires editors and reviewers to disclose conflicts of interest and to decline handling or reviewing manuscripts for which they may have a conflict of interest. The editors and reviewers of this article have no conflicts of interest.

Received for publication Aug 13, 2020; revisions received Aug 13, 2020; accepted for publication Aug 14, 2020; available ahead of print Aug 19, 2020.

Address for reprints: Muralidhar Padala, PhD, Cardiothoracic Research Laboratories, Emory University School of Medicine, 380B Northyards Blvd, Atlanta, GA 30313

(E-mail: spadala@emory.edu).

JTCVS Techniques 2020;4:138-9

2666-2507

Copyright (c) 2020 The Authors. Published by Elsevier Inc. on behalf of The American Association for Thoracic Surgery. This is an open access article under the CC BY-NCND license (http://creativecommons.org/licenses/by-nc-nd/4.0/).

https://doi.org/10.1016/j.xjtc.2020.08.041

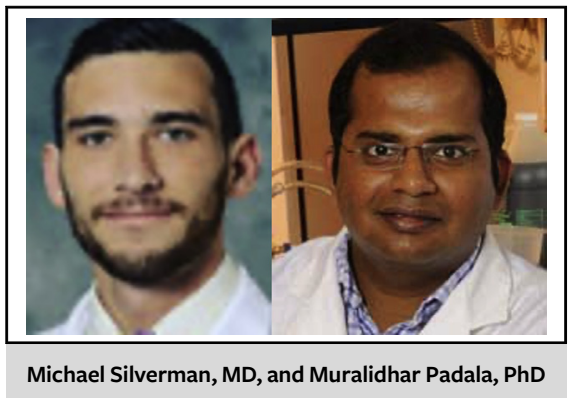

\section{CENTRAL MESSAGE \\ The basic tenet of mitral repair for ischemic mitral regurgitation is to relieve the ventricular teth- ering forces and restore coapta- tion. The proposed multiloop papillary muscle hoisting chord can be a valuable repair tech- nique for this lesion.}

reasonably understood that a durable repair that preserves the native mitral valve will always trump a replacement that is prone to structural deterioration in the mitral position. Surgical repair of IMR has largely focused on undersizing the mitral annulus, as in a majority of patients it is dilated. Because a larger annulus for a fixed leaflet area can restrict their coaptation, undersizing the annulus to a size that is lesser than the native annular dimensions is deemed an appropriate method. Although effective in most patients initially, and some patients in the long term, recurrence of mitral regurgitation is a frequent complication in most patients. Reducing the annulus with a ring draws the mitral leaflets into coaptation, but does not relieve the tethering forces imposed by the ventricle, leading to such poor durability. ${ }^{3}$

Concomitant subvalvular techniques to relieve tethering forces have been proposed for few decades, but none has entered into mainstream practice. Chordal cutting was among the first techniques proposed; a solution that was technically simple ${ }^{4}$ but mechanistically suboptimal due to the risky redistribution of forces it entails and ventricular dysfunction it causes. ${ }^{5,6}$ Papillary muscle approximation was proposed by Hvass and colleagues, ${ }^{7}$ and it has adequate long-term data and is increasingly being adopted into clinical practice. Papillary muscle hoisting or relocation is another approach proposed by Kron and colleagues, ${ }^{8}$ in which a 3-0 prolene suture is used to relocate the 
posteromedial papillary muscle toward the mitral annulus. ${ }^{8}$ The suture is passed twice through the fibrous portion of the posterior papillary muscle (without pledgets) and anchored onto the atrial surface of the mitral annulus before the annuloplasty. Short-term feasibility was excellent, but desired tethering of the papillary muscle without it twisting was not reproducible.

Torkan and colleagues ${ }^{9}$ report their experience with a modification of this original technique in 3 patients. In the video accompanying this work, the authors demonstrate the preparation of a 2-part polytetrafluoroethylene suture system for papillary muscle relocation. A first loop with 2 pledgets acts as a papillary muscle anchor, and multiple polytetrafluoroethylene sutures that loop into this anchor are drawn through the posterior aspect of the mitral annulus to hoist or relocate the papillary muscle. Implantation of this loop is performed before annuloplasty, but their tightening and knotting is performed after annuloplasty with a saline test to ensure desired coaptation. Quantitative data from the 3 patients are gleaned from preoperative and postoperative transesophageal imaging, and transthoracic imaging after 3 months of followup. End diastolic volume and end systolic volume are both decreased, and ejection fraction was increased. The short axis diameters of the chambers were unaltered, but volumes were significantly reduced, indicating a potential longitudinal remodeling of the heart. Considering the very small sample size, these functional data should be considered with that limitation in mind.

The authors should be congratulated for a logical improvement of the Kron procedure, with 2 distinct advantages that their modification allows: the pledgets can buttress the papillary muscle and reduce the risk of tearing, and the use of multiple loops allows for a tailored repair for different tethering anatomies, and provides a balanced hoisting of the muscle that avoids twisting of the muscle. The technical complexity of this approach is no greater than neochordoplasty, which is a widely adopted technique, and thus the approach has benefits. Performing this technique may be easier when approached via the Waterstone's groove or via the interatrial septum, rather than a left atrial approach. Thus, minimally invasive surgery via a right thoracotomy or a robotic mitral valve surgeon may be best suited to apply this technique.

A criticism of this study is the use of extensive downsizing of the mitral annulus, despite the subannular repair, which defeats the purpose. By relieving the tethering forces with papillary muscle hoisting, the authors could have achieved a better coaptation geometry by true-sizing the annulus than by downsizing it. As is evident from the video, extensive downsizing led to a restricted posterior leaflet, which can be improved in the future by selecting a ring that is larger. Another limitation is that a technique such as this would require better distension of the left ventricle than can be provided by a saline test because the end systolic volume when the heart is reanimated will be greater than the saline-filled arrested heart. An approach worth pursuing in this scenario is to use a pump to fill the heart until it is distended to a larger volume, and the chords are adjusted to tailor the coaptation. Altogether, this technique is a good addition to literature.

\section{References}

1. Levine RA, Hagege AA, Judge DP, Padala M, Dal-Bianco JP, Aikawa E, et al Mitral valve disease-morphology and mechanisms. Nat Rev Cardiol. 2015;12: 689-710.

2. Asgar AW, Mack MJ, Stone GW. Secondary mitral regurgitation in heart failure: pathophysiology, prognosis, and therapeutic considerations. J Am Coll Cardiol. 2015;65:1231-48.

3. Acker MA, Parides MK, Perrault LP, Moskowitz AJ, Gelijns AC, Voisine P, et al Mitral-valve repair versus replacement for severe ischemic mitral regurgitation. $N$ Engl J Med. 2014:370:23-32.

4. Messas E, Guerrero JL, Handschumacher MD, Conrad C, Chow CM, Sullivan S, et al. Chordal cutting: a new therapeutic approach for ischemic mitral regurgitation. Circulation. 2001;104:1958-63.

5. Padala M, Gyoneva L, Yoganathan AP. Effect of anterior strut chordal transection on the force distribution on the marginal chordae of the mitral valve. J Thorac Cardiovasc Surg. 2012;144:624-33.

6. Moon MR, DeAnda A Jr, Daughters GT II, Ingels NB, Miller DC. Effects of chordal disruption on regional left ventricular torsional deformation. Circulation 1996;94(9 Suppl):II143-51.

7. Hvass U, Tapia M, Baron F, Pouzet B, Shafy A. Papillary muscle sling: a new functional approach to mitral repair in patients with ischemic left ventricular dysfunction and functional mitral regurgitation. Ann Thorac Surg. 2003;75:809-11.

8. Kron IL, Green GR, Cope JT. Surgical relocation of the posterior papillary muscle in chronic ischemic mitral regurgitation. Ann Thorac Surg. 2002;74:600-1.

9. Torkan L, Servito MH, Bisleri G. Papillary muscle relocation with a multiloop suture: a proposed surgical technique for ischemic mitral regurgitation. J Thorac Cardiovasc Surg Tech. 2020;4:133-5. 